\title{
Avaliação da dieta habitual de escolares de Piracicaba: aplicação do Questionário de Freqüência Alimentar para Adolescentes (QFAA) reestruturado em estudo-piloto
}

\author{
Cristina Akemi Momoㄹ; Marina Bueno do Carmo ${ }^{1}$; Priscila Maria Fúncia Fernandez ${ }^{2}$; Silvia Maria Voci ${ }^{2}$; \\ Betzabeth Slater ${ }^{3}$; Marina Vieira da Silva ${ }^{4}$
}

O Questionário de Freqüência Alimentar para Adolescentes (QFAA), validado em amostra de adolescentes do município de São Paulo, passou por adaptação e reestruturação, para ser aplicado em Piracicaba. Objetivou-se aplicar o QFAA reformulado e descrever a dieta habitual de um grupo de escolares de Piracicaba. Após as modificações quanto ao número de itens alimentares e o tamanho das porções, o QFAA foi testado em 19 escolares com idade entre 10 e 14 anos para avaliar o consumo alimentar. Além de análises descritivas, o teste de Kolmogorov-Smirnov foi aplicado para todas as variáveis quantitativas contínuas. $\mathrm{O}$ teste $T$ de Student foi aplicado para comparação de médias entre os gêneros. Apenas para as variáveis que apresentaram variâncias desiguais aplicou-se o teste U de Mann-Whitney. O consumo médio habitual observado foi de 2803,30 kcal. As médias dos macronutrientes, ajustados pela ingestão calórica, foram de 93,16g para proteína, 107,33g para lipídios e 391,85g para carboidratos. As médias de porções consumidas por dia variaram de 1,07 (feijões) a 3,36 (doces). Observou-se um elevado consumo de doces (aproximadamente $113 \mathrm{~g} /$ dia) e reduzida ingestão de frutas e hortaliças quando comparados ao Guia Alimentar Americano. Não foram observadas diferenças significativas entre os gêneros para as variáveis deste estudo.

Palavras-chave: adolescentes, consumo alimentar, Questionário de Freqüência Alimentar

\section{Assessment of adolescents' usual intake from Piracicaba: use of Food Frequency Questionnaire for Adolescents (AFFQ) after re- structuration in a pilot study}

The Food Frequency Questionnaire for Adolescents (AFFQ), validated in a teenagers' sample in São Paulo city, was adapted and re-structured for its application in Piracicaba. The objective was to apply the reformulated instrument and describe the habitual intake of this sample. After modifications regarding the number of food items and portion sizes, the AFFQ was tried in 19 adolescents of 10 to 14 years of age in order to assess their food intake. Beyond descriptive analyses, the Kolmogorov-Smirnov's test was applied for all the continuous quantitative variables. The Student-T test was applied to compare averages between genders. Just for the variables that presented unlike variances, the Mann-Whitney U test was applied. The mean of usual intake observed was $2803.30 \mathrm{kcal}$. The adjusted macronutrients values were: $93.16 \mathrm{~g}$ for protein, $107.33 \mathrm{~g}$ for total fat and 391.85g for carbohydrates. Mean daily portions eaten ranged from 1.07 (beans) to 3.36 (sweets). It is concluded that a high intake of sweets (approximately 113g) and a reduced intake of fruits and vegetables were practiced by these youngsters when compared with the American Food Guide. Statistically significant differences haven't been observed among variables of this study.

Keywords: Adolescents, dietary intake, Food Frequency Questionnaire

\footnotetext{
1 Bolsista de Iniciação Científica do Conselho Nacional de Desenvolvimento Científico e Tecnológico (CNPq) do Departamento de Nutrição da Faculdade de Saúde Pública da USP CNPq nº. processos 111216/2003-6 e 111929/2004-0

2 Mestranda do Departamento de Nutrição da Faculdade de Saúde Pública da USP

3 Professora Doutora do Departamento de Nutrição da Faculdade de Saúde Pública da USP

4 Professora Doutora do Departamento de Agroindústria, Alimentos e Nutrição da Escola Superior de Agricultura Luiz de Queiroz/
} 


\section{Introdução}

A adolescência consiste em um período de transição entre a infância e a vida adulta, caracterizado por intensas mudanças somáticas, psicológicas e sociais, compreendendo o estágio de vida entre 10 e 19 anos de idade ${ }^{[1]}$. Nesse período da vida vários fatores estão relacionados com o comportamento alimentar, tais como aspectos nutricionais, demográficos, socioeconômicos, culturais, ambientais e psicológicos ${ }^{[2]}$.

Para uma correta avaliação do consumo alimentar, faz-se necessária a adoção de instrumentos adequados, de preferência validados na mesma população ${ }^{[3]}$.

Os Questionários de Freqüência Alimentar (QFA) constituem importante recurso para a epidemiologia de doenças crônicas, na medida em que viabilizam a avaliação do consumo alimentar habitual e, conseqüentemente, de fatores dietéticos de exposição de longo período. A sua utilização tem sido progressivamente mais expressiva, tornando-se o método dominante para avaliação do consumo alimentar em estudos de caso-controle e coorte ${ }^{[4,5,6]}$.

Esse instrumento é delineado para obter informações qualitativas e quantitativas da ingestão alimentar de um indivíduo, a partir de um formato pré-estruturado, por meio de uma lista de alimentos e alternativas de freqüência de consumo para cada item ${ }^{[7,8]}$.

Contudo, é necessário considerar que a eficácia desse instrumento depende de vários fatores, tais como a seleção e o agrupamento dos alimentos que integram o questionário e o conhecimento da porção média habitualmente ingerida, que podem modificar as estimativas da ingestão de determinados constituintes alimentares ${ }^{[6]}$.

Embora o Questionário de Freqüência Alimentar para Adolescentes (QFAA) desenvolvido por SLATER et al. ${ }^{[9]}$ tenha sido validado e tenha revelado desempenho razoável, foram necessárias: a) sua reestruturação gráfica; b) modificações quanto ao tamanho de porções de alimentos; c) sua adaptação, tendo em vista que a faixa etária e o consumo alimentar dos indivíduos que integrariam a amostra são diferentes daqueles da população da validação.

O estudo teve como objetivo aplicar e testar previamente o QFAA reformulado e, a partir dos dados obtidos, descrever a dieta habitual de um grupo de escolares de Piracicaba, com idade entre 10 e 14 anos, de ambos os sexos.

\section{Metodologia}

\section{Estrutura inicial do QFAA}

A lista de alimentos do QFAA foi elaborada a partir de dados de um estudo realizado com adolescentes com idade entre 10 e 19 anos, avaliados por dois diários alimentares ${ }^{[10]}$. A relação de alimentos foi resumida a 140 itens agrupados segundo suas características físicas e perfil de nutrientes. A partir desta segunda lista, foram identificados os alimentos de maior contribuição energética, usando-se a técnica de análise estatística de proporção ponderada utilizada por BLOCK et al. ${ }^{[11]}$, HOWE et al. ${ }^{[12]}$ e COLUCCI et al. ${ }^{[13]}$ As porções referidas no instrumento também foram obtidas a partir do mesmo banco de dados e definidas de acordo com a metodologia proposta pelos referidos autores. A maioria das porções expressa o consumo médio em gramas para cada item alimentar ou unidades de alimento. A versão final do QFAA contava com 76 itens alimentares.

\section{Reestruturação do QFAA}

Para a reestruturação do QFAA foi utilizado o banco de dados do estudo de MANTOANELLI ${ }^{[14]}$. Neste estudo, o QFAA foi aplicado em estudantes de uma escola da cidade de São Paulo (SP), tendo sido identificados novos alimentos e tamanhos de porções. Dessa maneira, foram incluídos no QFAA os alimentos que apresentaram elevada freqüência de consumo e que não integravam a lista da versão validada. A versão reestruturada do QFAA foi composta por 94 alimentos, não sofrendo alterações nas freqüências estabelecidas no estudo de validação. Essa reformulação visou complementar o instrumento com itens substancialmente consumidos pela população adolescente, na tentativa de coletar informações mais completas e acuradas.

A forma gráfica do questionário foi modificada para facilitar seu preenchimento. $\mathrm{O}$ formato vertical e a exclusão da totalidade das figuras reduziram a extensão do instrumento. Além disso, para facilitar a construção do banco de dados, foi adicionada uma coluna para a codificação das freqüências. 
Após as modificações, tanto na estrutura impressa como na eletrônica (para digitação dos dados), foi necessária a aplicação do instrumento em população que reunisse características semelhantes.

\section{População de estudo}

Participaram deste estudo 19 adolescentes voluntários, com idade entre 10 e 14 anos, de ambos os sexos, matriculados nas $5^{a}, 6^{a}$ e $7^{a}$ séries de uma escola da rede pública de ensino de Piracicaba, São Paulo. Foi utilizado, como critério de inclusão o consumo de energia entre $500 \mathrm{kcal}$ e $6000 \mathrm{kcal}^{[15]}$, excluindo-se, dessa forma, dois indivíduos do sexo feminino e um por preenchimento incompleto do QFAA, totalizando uma amostra de 16 adolescentes.

Foram analisados os dados antropométricos (peso e altura), idade, sexo e variáveis dietéticas (energia, carboidrato, proteína, lipídio total, fibra, colesterol, retinol, vitamina $\mathrm{C}$, cálcio e ferro).

\section{Coleta e processamento de dados}

As entrevistas e a aferição das medidas antropométricas foram realizadas por pesquisadores e estudantes universitários da área de nutrição e alimentos, previamente treinados.

O estado nutricional foi avaliado por meio do cálculo do Índice de Massa Corporal (IMC), expresso pela seguinte fórmula:

$$
I M C=\frac{\operatorname{peso}(\mathrm{Kg})}{\operatorname{altura}^{2}(\mathrm{~m})}
$$

Posteriormente, os adolescentes foram classificados a partir das curvas de IMC por idade do NCHS ${ }^{[16]}$.

Os entrevistadores receberam um treinamento específico e um manual de instruções, elaborado para a correta aplicação e preenchimento do QFAA. As informações dietéticas foram coletadas por meio de entrevista pessoal, pela utilização do QFAA reestruturado, incluindo questões sobre a freqüência usual de consumo nos últimos seis meses.

O processamento dos dados do QFAA foi feito no programa DIETSYS ${ }^{[17]}$. Para a inclusão dos novos alimentos nesse software, foi necessária a verificação de seus valores nutricionais, por meio de consulta à tabela de composição nutricional do USDA ${ }^{[18]}$, no programa Virtual Nutri ${ }^{[19]}$ e em rótulos de alimentos industrializados. Antes de serem inseridas no programa, as preparações foram desmembradas em ingredientes, e o valor nutricional das porções foi calculado utilizando-se respectivamente o "Manual de receitas e medidas caseiras para cálculos de inquéritos alimentares" [20] e a "Tabela para avaliação de consumo alimentar em medidas caseiras" ${ }^{[21]}$. Os alimentos que não foram encontrados nas citadas referências foram pesados no Laboratório de Técnica Dietética da Faculdade de Saúde Pública da Universidade de São Paulo.

Para a comparação dos dados de consumo alimentar, utilizou-se o Guia Alimentar Americano ${ }^{[22]}$, e as porções registradas no DASH Eating Plan [23] foram utilizadas para o grupo referente ao consumo de açúcares e doces.

Para o processamento e análise dos dados, foram utilizados os programas Stattistical Package for the Social Sciences - versão $10^{[24]}$ e Excel para Windows 2003.

Foram elaboradas análises descritivas dos dados, teste $t$-student para comparação de médias entre os sexos (para as variáveis que apresentavam igualdade de variâncias) e o teste não-paramétrico $U$ de MannWhitney, para comparação de médias de variáveis que não apresentavam todos os pressupostos para o teste t-student.

\section{Resultados}

Após a aplicação do QFAA em estudopiloto, verificou-se a necessidade de efetuar novas modificações em sua estrutura, como a inclusão de preparação típica da região (pamonha). Também foram alterados os itens relativos aos sucos de frutas naturais. $\mathrm{Na}$ versão anterior ao teste, os sucos eram apresentados com e sem adição de açúcar. Após o estudo-piloto, verificou-se a baixa freqüência de consumo de sucos não-adoçados, optando-se pela sua exclusão. No caso de algum alimento, ou preparação, consumido e não-constante da listagem, este era adicionado na parte aberta a essas observações.

A média de idade dos alunos que participaram do estudo foi de 12 anos, sendo que a maioria era do sexo feminino $(n=9 ; 56,3 \%)$. 
Os valores médios de peso, altura e IMC, segundo o sexo, são apresentados na tabela 1 , sendo que as médias do grupo foram de 48,21 Kg, 1,56m e $19,63 \mathrm{Kg} / \mathrm{m}^{2}$.

A partir do IMC, o estado nutricional foi classificado, observando-se que a maioria dos adolescentes estava eutrófica. Dos 16 indivíduos, 3 apresentavam excesso de peso $(19 \%)$.

A tabela 2 apresenta os valores médios de energia e nutrientes validados e avaliados pelo QFAA, tanto brutos quanto ajustados pela energia. Observa-se que, para a maioria dos nutrientes, a significância do teste melhora à medida que se trabalha com os valores ajustados, exceto para cálcio, vitamina $\mathrm{C}$, fibra e retinol. As médias dos nutrientes (exceto o colesterol) e energia foram maiores para o sexo masculino.

A tabela 3 apresenta os valores dos macronutrientes (em porcentagem em relação ao valor calórico total médio da amostra), na qual se pode destacar a porcentagem de lipídios acima de 30\%.

A tabela 4 apresenta o número de porções consumidas por dia, de cada grupo alimentar e seus respectivos valores em gramas, de acordo com o sexo. Observa-se o reduzido consumo de frutas e hortaliças nessa população, bem como dos alimentos integrantes do grupo do leite e derivados. Em contrapartida, o grupo de alimentos mais consumido diariamente (aproximadamente 113g) foi o de doces, que inclui chocolates, bolos, sorvetes, achocolatados, açúcar de adição, balas, doces de frutas, sobremesas do tipo mousse e biscoitos recheados. Para o sexo feminino, o único grupo de alimentos que apresentou valor superior, quando comparado aos dados obtidos para os meninos, foi o das carnes, condizendo com os valores mais elevados de colesterol e porcentagem de proteínas, em relação à energia total (Tabela 3).
O grupo dos feijões apresentou médias de porções muito semelhantes para ambos os gêneros, com consumo em torno de uma porção diária.

Não foram observadas diferenças estatisticamente significativas entre os gêneros para todas as variáveis estudadas.

\section{Discussão}

No estudo de validação do QFAA desenvolvido por SLATER et al. ${ }^{[9]}$, o instrumento obteve bom desempenho em relação à acurácia, apresentando uma precisão aceitável para classificar os indivíduos segundo seu consumo de energia e da maioria dos nutrientes (exceto para retinol e ferro), sendo que essa precisão se revela distinta quando se discriminam os indivíduos por sexo.

O QFAA foi aplicado neste estudo-piloto, em amostra distinta da população utilizada para sua validação, sendo submetido posteriormente a um processo de calibração.

A maioria dos adolescentes integrantes da pesquisa revelou estado nutricional adequado, sendo que apenas dois indivíduos possuíam peso acima do normal. Quando comparados os dados de peso, altura e IMC com o estudo realizado por GARCIA et al. ${ }^{[25]}$, observam-se valores superiores, e média semelhante para o IMC, em ambos os sexos, não se apresentando diferenças significativas. Quando são considerados os dados obtidos por CARVALHO et al. ${ }^{[26]}$, tendo por base amostra de adolescentes com idade entre 10 e 19 anos, observa-se que as médias de peso e altura se revelam muito próximas às obtidas com a população estudada nesta pesquisa.

Em estudo realizado por BULCK e MIERLO [27], foram avaliados adolescentes matriculados no

Tabela 1. Média, desvio-padrão e valores de $p$ das variáveis antropométricas, de acordo com o gênero de adolescentes de escola pública, Piracicaba, 2004.

\begin{tabular}{lcccc}
\hline Variáveis & Total & \multicolumn{2}{c}{ Sexo } & $P$ \\
\cline { 3 - 4 } & & Masculino & Feminino & \\
\hline Peso & $48,21(9,64)$ & $48,21(12,27)$ & $48,20(7,83)$ & 0,59 \\
Altura & $1,56(0,09)$ & $1,57(0,11)$ & $1,56(0,07)$ & 1,00 \\
IMC & $19,63(2,61)$ & $19,39(2,87)$ & $19,82(2,55)$ & 0,83 \\
\hline
\end{tabular}


Tabela 2. Média, desvio-padrão e valores de $p$ de energia e nutrientes brutos e ajustados, de acordo com o gênero de adolescentes de escola pública, Piracicaba, 2004.

\begin{tabular}{|c|c|c|c|c|}
\hline \multirow{2}{*}{$\begin{array}{l}\text { Energia e } \\
\text { nutrientes }\end{array}$} & \multirow[t]{2}{*}{$\operatorname{Total}(D P)$} & \multicolumn{2}{|c|}{ Sexo } & \multirow[t]{2}{*}{$P$} \\
\hline & & Masculino(DP) & $\operatorname{Feminino}(D P)$ & \\
\hline Energia (Kcal) & $2803,30(1361,63)$ & $3227,61(1534,68)$ & $2473,28(1195,74)$ & 0,29 \\
\hline \multicolumn{5}{|l|}{ Proteína (g) } \\
\hline Bruto & $91,97(52,94)$ & $98,30(52,78)$ & $87,04(55,71)$ & 0,69 \\
\hline Ajustado & $93,16(17,95)$ & $83,97(10,96)$ & $100,31(19,57)$ & 0,07 \\
\hline \multicolumn{5}{|l|}{ Lipídios (g) } \\
\hline Bruto & $107,79(63,58)$ & $122,59(72,44)$ & $96,27(57,48)$ & 0,43 \\
\hline Ajustado & $107,33(16,15)$ & $102,96(17,51)$ & $110,72(15,16)$ & 0,36 \\
\hline \multicolumn{5}{|l|}{ Carboidrato (g) } \\
\hline Bruto & $391,26(176,39)$ & $461,78(195,31)$ & $336,42(148,31)$ & 0,17 \\
\hline Ajustado & $391,85(42,16)$ & $408,99(40,18)$ & $378,52(40,80)$ & 0,16 \\
\hline \multicolumn{5}{|l|}{ Cálcio (mg) } \\
\hline Bruto & $824,59(482,02)$ & $988,30(499,76)$ & $697,27(454,58)$ & 0,24 \\
\hline Ajustado & $824,26(222,28)$ & $854,68(235,38)$ & $800,60(222,83)$ & 0,65 \\
\hline \multicolumn{5}{|l|}{ Ferro (mg) } \\
\hline Bruto & $17,07(8,40)$ & $20,57(10,05)$ & $14,35(6,12)$ & $0,15^{*}$ \\
\hline Ajustado & $17,65(2,88)$ & $18,69(3,76)$ & $16,84(1,80)$ & 0,21 \\
\hline \multicolumn{5}{|c|}{ Vitamina C (mg) } \\
\hline Bruto & $137,99(98,90)$ & $184,07(124,25)$ & $102,15(58,81)$ & 0,10 \\
\hline Ajustado & $138,85(60,09)$ & $160,45(73,34)$ & $122,04(44,83)$ & 0,22 \\
\hline \multicolumn{5}{|l|}{ Colesterol (mg) } \\
\hline Bruto & $251,11(182,36)$ & $249,80(147,58)$ & $252,13(214,51)$ & 0,98 \\
\hline Ajustado & $250,69(111,19)$ & $204,34(48,26)$ & $286,74(134,51)$ & $0,22^{*}$ \\
\hline \multicolumn{5}{|l|}{ Fibra (g) } \\
\hline Bruto & $21,43(11,27)$ & $25,00(13,65)$ & $18,65(8,86)$ & 0,28 \\
\hline Ajustado & $22,70(4,62)$ & $23,06(3,47)$ & $22,41(5,55)$ & 0,79 \\
\hline \multicolumn{5}{|l|}{ Retinol (mg) } \\
\hline Bruto & $592,82(359,44)$ & $751,24(392,73)$ & $469,59(295,85)$ & 0,12 \\
\hline Ajustado & $591,55(286,46)$ & $682,32(329,45)$ & $520,95(244,21)$ & 0,28 \\
\hline
\end{tabular}

* nível de significância para o teste não paramétrico $U$ de Mann-Whitney 
Tabela 3. Participação de macronutrientes no Valor Energético Total, de acordo com o gênero de adolescentes de escola pública, Piracicaba, 2004.

\begin{tabular}{lccc}
\hline $\begin{array}{l}\text { Macronutrientes } \\
\text { (em gramas) }\end{array}$ & Total $(\%)$ & \multicolumn{2}{c}{ Sexo } \\
\cline { 3 - 4 } & & Masculino & Feminino \\
\hline Peso & 12,7 & 11,8 & 13,6 \\
Altura & 33,4 & 33,0 & 33,8 \\
IMC & 53,9 & 55,2 & 52,6 \\
\hline
\end{tabular}

primeiro ano do ensino secundário, com média de idade de 13,2 anos. Foi possível observar que as médias de peso, de acordo com o sexo, foram inferiores, porém a altura e o IMC apresentaram semelhança em relação às médias obtidas para os adolescentes da presente pesquisa.

EPSTEIN et al. ${ }^{[28]}$ analisaram dados de adolescentes, com idade entre 12 e 16 anos, e identificaram valores de altura e peso superiores para os meninos e semelhantes para as meninas. Quanto ao IMC, os valores foram semelhantes para ambos os sexos.

Em relação ao consumo alimentar, comparandose os dados do presente estudo, a quantidade de energia descrita por EPSTEIN et al. [28], a partir da aplicação de três Recordatórios de 24 horas (R24h), foi inferior para os meninos e muito semelhante para as meninas, sendo que o mesmo pode ser observado no estudo realizado no Rio de Janeiro por SICHIERI [29], que analisou os dados provenientes de QFA de adolescentes com idade entre 12 e 14 anos.

Comparando-se os resultados obtidos por STOCKMAN et al. ${ }^{[30]}$, por meio de três registros alimentares aplicados em adolescentes com idade entre 14 e 18 anos, aos dados obtidos neste trabalho, notouse consumo inferior de energia $(2624 \mathrm{kcal})$, lipídios $(96,0 \mathrm{~g})$, carboidratos $(343,3 \mathrm{~g})$, ferro $(15,5 \mathrm{mg})$ e fibras $(14,0 \mathrm{~g})$, e superior de proteínas $(96,8 \mathrm{~g})$, colesterol (273,5mg) e cálcio (1022,0mg), não ajustados pela energia. No estudo de SICHIERI ${ }^{[29}$ também se pôde notar que o consumo para os mesmos nutrientes e para a vitamina $C$ foi superior para ambos os sexos. No entanto, em relação ao consumo de carboidratos e ferro, os valores observados foram inferiores para os meninos ( $437 \mathrm{~g}$ e $17,7 \mathrm{mg}$, respectivamente) e superiores para as meninas (412g e 16,1 mg).
No estudo realizado por GARCIA et al. ${ }^{[25]}$, foram avaliados adolescentes com idade entre $10 \mathrm{e}$ 14 anos, por meio de R24h, sendo que os valores de energia, macronutrientes, cálcio e ferro foram bem inferiores aos observados neste estudo. Somente o colesterol apresentou valor superior. Vale ressaltar que a metodologia aplicada no estudo de GARCIA et al. ${ }^{[25]}$ incluiu a aplicação de apenas um R24h, o que não é suficiente para caracterizar a dieta habitual.

Ainda no tocante ao consumo de energia, ALBANO et al. ${ }^{[31]}$, LERNER et al. ${ }^{[32]}$ e NUZZO ${ }^{[10]}$ observaram valores inferiores ao analisar dados relativos a adolescentes brasileiros de ambos os sexos. Esses estudos tiveram por base amostra de indivíduos cuja idade variou entre 10 e 18 anos.

No estudo de SICHIERI ${ }^{[29]}$, foram observados valores percentuais de lipídios na dieta inferiores aos apresentados na tabela 3, sendo $25,4 \%$ para indivíduos do sexo masculino e de $28,0 \%$ para o feminino.

Assim como neste estudo, não foi observada por GARCIA et al. ${ }^{[25]}$, para médias de consumo de energia e nutrientes, exceto cálcio, diferença estatisticamente significativa para os sexos. BULCK e MIERLO [27], porém, observaram diferenças significativas em relação ao consumo de energia, que se revelou superior para os meninos.

SPECK et al. ${ }^{[33]}$ também não observaram diferenças significativas entre as médias de consumo de energia e porções de alimentos, de acordo com o sexo. Deve-se considerar que o questionário de freqüência utilizado pelos referidos autores avaliou apenas o período de uma semana pregressa. Os entrevistados foram questionados sobre o consumo de alimentos com freqüência de um dia por semana, incluindo também questões sobre hábitos alimentares 
Tabela 4. Média, desvio-padrão e valores de $p$ dos grupos de alimentos representados em porções/dia e gramas, conforme o gênero de adolescentes de escola pública, Piracicaba, 2004.

\begin{tabular}{|c|c|c|c|c|}
\hline \multirow[t]{2}{*}{ Grupos alimentares } & \multirow[t]{2}{*}{ Total(DP) } & \multicolumn{2}{|c|}{ Sexo } & \multirow[t]{2}{*}{$P$} \\
\hline & & Masculino(DP) & Feminino(DP) & \\
\hline \multicolumn{5}{|l|}{ Frutas } \\
\hline \multicolumn{5}{|l|}{ Porções } \\
\hline Gramas & $1,25(0,88)$ & $1,44(0,94)$ & $1,10(0,86)$ & 0,46 \\
\hline Hortaliças & $138,33(98,71)$ & $164,53(106,82)$ & $117,96(92,97)$ & 0,37 \\
\hline \multicolumn{5}{|l|}{ Porções } \\
\hline Gramas & $1,79(1,92)$ & $2,20(2,37)$ & $1,48(1,56)$ & 0,47 \\
\hline Doces & $207,59(205,80)$ & $262,13(245,67)$ & $165,17(171,82)$ & 0,37 \\
\hline \multicolumn{5}{|l|}{ Porções } \\
\hline Gramas & $3,36(1,80)$ & $3,67(1,74)$ & $3,11(1,91)$ & 0,55 \\
\hline Feijões & $113,18(53,82)$ & $123,13(53,80)$ & $105,44(55,74)$ & 0,53 \\
\hline \multicolumn{5}{|l|}{ Porções } \\
\hline Gramas & $1,07(0,71)$ & $1,04(0,72)$ & $1,09(0,74)$ & 0,90 \\
\hline Leite e derivados & $91,36(61,19)$ & $89,50(61,73)$ & $92,81(64,47)$ & 0,92 \\
\hline \multicolumn{5}{|l|}{ Porções } \\
\hline Gramas & $1,66(1,40)$ & $1,84(1,19)$ & $1,52(1,60)$ & 0,67 \\
\hline Carnes & $276,03(220,74)$ & $348,23(221,99)$ & $219,87(215,15)$ & 0,26 \\
\hline \multicolumn{5}{|l|}{ Porções } \\
\hline Gramas & $1,31(1,24)$ & $1,01(0,89)$ & $1,54(1,47)$ & 0,42 \\
\hline $\begin{array}{l}\text { Cereais, pães e } \\
\text { massas }\end{array}$ & $100,08(96,78)$ & $77,57(70,26)$ & $117,59(114,33)$ & 0,43 \\
\hline \multicolumn{5}{|l|}{ Porções } \\
\hline \multirow[t]{2}{*}{ Gramas } & $2,74(1,19)$ & $3,23(0,96)$ & $2,36(1,26)$ & 0,15 \\
\hline & $296,34(134,18)$ & $338,54(102,53)$ & $263,51(151,99)$ & 0,28 \\
\hline
\end{tabular}

* nível de significância para o teste não paramétrico U de Mann-Whitney

relacionados, por exemplo, ao uso de sal.

No estudo de EPSTEIN et al. ${ }^{[28]}$, somente foi observada diferença no consumo de proteínas, sendo que a ingestão para meninos foi significativamente maior. Quando observada a ingestão de proteína, após ajuste pela energia, a diferença aproximou-se da significância $(p=0,07)$, indicando uma tendência para maior consumo desse nutriente entre as meninas, contrariamente ao observado por EPSTEIN et al. ${ }^{[28]}$, em amostra com o mesmo número de indivíduos, porém de faixa etária diferente. Vale ressaltar que as faixas etárias de 10 a 14 anos e 12 a 16 anos guardam uma diferença no que se refere, principalmente, aos estágios de maturação sexual.

DEMORY-LUCE et al. ${ }^{[34]}$ descreveram o consumo de grupos alimentares em gramas, utilizando dados do Bogalusa Heart Study, realizado com americanos de ascendência africana e européia. A ingestão foi levantada na década de 70 por meio de um R24h aplicado em indivíduos com 10 anos de idade e que foram reavaliados entre 1989 e 1991, quando foram considerados adultos jovens. 
O consumo diário dos indivíduos aos 10 anos de idade foi de $115,7 \mathrm{~g}$ de frutas e sucos, 139,6g de hortaliças, 182,9g de pães e grãos, $175 \mathrm{~g}$ de carnes, 137,9g de sobremesas e confeitos, 413,8g de leite e derivados. Comparando-se essas informações com os dados reunidos na Tabela 4, nota-se que os valores consumidos pelos adolescentes de Piracicaba foram inferiores para os grupos de doces, leite e derivados e carnes, enquanto que, para frutas, hortaliças e pães, se revelaram superiores. Embora o consumo observado no estudo citado anteriormente não represente a ingestão habitual e se trate de um período muito pregresso, talvez parte das diferenças nos grupos de frutas e hortaliças possa ser decorrente do maior acesso e oferta desses itens no município de Piracicaba.

SICHIERI [29] identificou um consumo superior de feijão (124g) e leites e derivados, quando comparado ao encontrado nesta pesquisa. No entanto, no tocante às hortaliças, foi observado um consumo médio inferior $(175 \mathrm{~g})$.

Emestudorealizadocomadolescentesamericanos, cuja média de idade era de aproximadamente 13 anos, SPECK et al. ${ }^{[33]}$ observaram, por meio da utilização de Questionário de Hábitos Alimentares e adoção da Pirâmide Alimentar americana como parâmetro, um consumo médio de porções de 5,2 para pães e cereais, 1,6 para hortaliças, 2,6 para frutas, 6,0 para o grupo das carnes, 2,6 para leite e derivados e 5,7 para doces. Dessa maneira, todos os grupos observados apresentaram consumo superior quando comparados aos resultados obtidos com os alunos desta pesquisa (Tabela 4), exceto para hortaliças, cujo consumo se revelou muito semelhante.

No estudo de MAGAREY et al. ${ }^{[35]}$, o consumo de frutas em adolescentes australianos com idade entre 12 e 15 anos foi de 0,9 e 1,1 porções para meninos e meninas, respectivamente. Em relação ao consumo de hortaliças, os meninos consumiram cerca de 2,2 porções, enquanto as meninas consumiram 2,0 porções. Comparando-se com os dados obtidos, os meninos ingeriram mais frutas, sendo que as meninas apresentaram consumo igual aos dados citados. Quanto às hortaliças, as porções identificadas revelaram-se menores para ambos os sexos. O inquérito utilizado no estudo de MAGAREY et al. ${ }^{[35]}$ foi o R24h, representando a dieta atual da amostra.
No trabalho de CARVALHO et al. ${ }^{[26]}$, realizado em Teresina, Piauí, tendo como amostra indivíduos com idade entre 10 e 19 anos, o consumo de leguminosas verificado por meio do QFA foi semelhante ao encontrado por este estudo para o sexo feminino (1,19 porções). Para os meninos, o valor no referido estudo foi de 1,61 porções.

Quando comparado o consumo diário dos grupos alimentares avaliados com o Guia Alimentar para a população americana ${ }^{[2]}$ e aquele de acordo com a média de energia consumida pelos adolescentes, observou-se que, para os grupos de frutas, de hortaliças e de leite e derivados, o número de porções consumidas revelou-se inferior ao preconizado. Para o grupo de cereais, pães e massas e para o grupo de carnes somado ao de feijões, os valores identificados apresentam-se próximos ao considerado adequado. Como nas recomendações do USDA ${ }^{[22]}$ não existe parâmetro para número de porções de açúcares e doces, foi utilizado como ponto de corte as porções registradas no DASH Eating Plan ${ }^{[23]}$, observando-se, dessa maneira, que a quantidade consumida pelo grupo de alunos de Piracicaba foi mais elevada.

MAGAREY et al. ${ }^{[35]}$, avaliando indivíduos de faixa etária de 12 a 15 anos, identificaram diferenças entre os gêneros no tocante ao consumo de porções de alimentos.

A não observação de diferenças em relação ao gênero no presente estudo pode ser atribuída também ao tamanho da amostra, sendo que, na maioria dos estudos citados, o número de indivíduos era superior.

Embora nos estudos de EPSTEIN et al. ${ }^{[28]}$, STOCKMAN et al. ${ }^{[30]}$, LERNER et al. ${ }^{[32]}$ e NUZZO ${ }^{[10]}$ tenham sido adotados os métodos do R24h e registros alimentares para avaliação de consumo, a aplicação desses instrumentos em mais de um momento garante dados da dieta habitual que guardam relação com os dados obtidos por QFA, mesmo considerando a exposição a diferentes tipos de erro peculiares a cada um deles.

Nos estudos de GARCIA et al. ${ }^{[25]}$, DEMORYLUCE et al. ${ }^{[34]}$ e MAGAREY et al. ${ }^{[35]}$, foi utilizado o R24h em apenas um momento de avaliação, não permitindo inferências no tocante à causalidade e definição de padrões alimentares, sendo possível 
apenas descrever o consumo alimentar atual.

\section{Conclusão}

No presente estudo, não foram observadas diferenças significativas entre os sexos para as variáveis antropométricas e de consumo, o que pode ser devido ao baixo número de integrantes na amostra.

A escassez de estudos envolvendo adolescentes, principalmente com idade entre 10 e 14 anos e que utilizem como instrumento de avaliação o QFA, dificulta a comparação dos dados. Por meio dos resultados obtidos, foi possível observar um consumo diário de lipídios acima de 30\% do valor calórico total. Notou-se também um elevado consumo de doces, de acordo com os pontos de corte já mencionados na discussão, para ambos os sexos.

Embora neste estudo se tenham estabelecido comparações entre o consumo e as porções registradas na Pirâmide Alimentar, mostrando a inadequação para a maioria dos grupos estudados, deve-se ressaltar que o parâmetro constitui um guia alimentar para a população norte-americana, com idade acima de 5 anos. Tal opção decorre do fato de não existir nenhum guia alimentar elaborado para a população brasileira que tenha obtido reconhecimento consensual e, ainda, que preconize valores para o grupamento etário de interesse desta pesquisa.

Vale ressaltar também que o tamanho amostral dos estudos mencionados é bastante distinto daquele que se vê neste, além do que nem todos utilizaram metodologias semelhantes para coleta de dados de consumo, comprometendo de certa forma a comparabilidade dos resultados obtidos.

É de fundamental importância a realização de estudos que monitorem os hábitos alimentares da população de adolescentes-utilizando-se instrumentos adequados -, bem como o desenvolvimento de pesquisas que subsidiem a elaboração de métodos que permitam a avaliação e representem o consumo desse grupo populacional com melhor acurácia.

Não foi possível testar o desempenho do QFAA, uma vez que não estavam disponíveis dados provenientes de métodos de referência (R24h) aplicados em mais de um momento. Cabe salientar também que após o estudo-piloto, novas modificações foram realizadas, tornando premente a necessidade de se testar seu desempenho após as alterações.

\section{Referências Bibliográficas}

[1] [OMS] Organización Mundial de la Salud. La salud de los jóvenes: un reto y una esperanza. Genebra: OMS; 1995.

[2] Fisberg M. Atualização em obesidade na infância e adolescência. São Paulo: Atheneu; 2004.

[3] Willett W, Lenart E. Reproducibility and validity of food-frequency questionnaires. In: Willett W. Nutritional epidemiology. 2. ed. New York: Oxford University Press; 1998.

[4] Drewnowski A. Diet image: a new perspective on the food-frequency questionnaire. Nutr Rev 2001; 59 (11): 370-2.

[5] Hoffmann K, Kroke A, Klipstein-Grobusch $\mathrm{K}$, Boeing H. Standardization of dietary intake measurements by nonlinear calibration using shortterm reference data. Am J Epidemiol 2002; 156 (9): 862-70.

[6] Moreira P, Sampaio D, Almeida MDV. Validade relativa de um questionário de freqüência de consumo alimentar através da comparação com um registro alimentar de quatro dias. Acta Médica Portuguesa 2003; 16 (6): 412-20.

[7] Food consumption of individuals. In: Gibson RS. Principles of nutritional assessment. New York: Oxford University Press; 1990.

[8] Armstrong BK, White E, Saracci R. Principles of exposure measurement in epidemiology. 2.ed. New York: Oxford University Press; 1995.

[9] Slater B, Philippi ST, Fisberg RM, Latorre MRDO. Validation of a semi-quantitative adolescent food frequency questionnaire applied at a public school in São Paulo, Brazil. Eur J Clin Nutr 2003; 57 (5): 629-35.

[10] Nuzzo, L. Avaliação do estado nutricional de adolescentes de uma escola privada de ensino [dissertação de mestrado]. São Paulo: Universidade de São Paulo; 1998.

[11] Block G, Dresser CM, Hartman AM, Carroll MD. 
Nutrient sources in the American diet: quantitative data from the NHANES II survey. II. Macronutrients and fats. Am J Epidemiol 1985; 122: 27-40.

[12] Howe GR, Harrison L, Jain M. A short diet history for assessing dietary exposure to $\mathrm{n}$-nitrosamines in epidemiological studies. Am J Epidemiol 1986; 124: 595-602.

[13] Colucci ACA, Nuzzo L, Slater B, Philippi ST. Energia na dieta dos adolescentes. In: Anais do $1^{\circ}$ Congresso Latino-Americano de Nutrição Humana; 1999, 24-27 jun. Gramado. Porto Alegre: Plenarium Ltda.; 1999. p. 34.

[14] Mantoanelli G. Dieta habitual de adolescentes de uma escola estadual do município de São Paulo [dissertação de mestrado]. São Paulo: Universidade de São Paulo; 2003.

[15] Andrade RG, Pereira RA, Sichieri R. Consumo alimentar de adolescentes com e sem sobrepeso do município do Rio de Janeiro. Cad Saúde Pública 2003; 19 (5): 1485-95.

[16] [CDC] Center of Disease Control and Prevention. National Center for Health Statistics. CDC growth charts 2000. Disponível em: < http://www.cdc.gov/ growthcharts >. Acesso em 5 mar. 2005.

[17] [NCI] HHHQ - Dietsys Analysis Software. Versão 4.01. National Cancer Institute, 1999.

[18] [USDA] United States Department of Agriculture. Nutritive Value of Foods.[on line]. Disponível em: $<$ http://www.nal.usda.gov/fnic/foodcomp $>$. Acesso em 30 mar. 2004 .

[19] Philippi ST, Szarfac SC, Latterza AR. Virtual Nutri (software). Versão 1.0 for Windows. São Paulo: Departamento de Nutrição da Faculdade de Saúde Pública da Universidade de São Paulo; 1996.

[20] Fisberg RM, Slater B. Manual de receitas e medidas caseiras para cálculos de inquéritos alimentares. São Paulo: Signus; 2002.

[21] Pinheiro ABV. Tabela para Avaliação de Consumo Alimentar em medidas caseiras. 5. ed. Rio de Janeiro: Atheneu; 2004.

[22] [USDA] United States Department of Agriculture.
Dietary Guidelines for Americans 2005. [on line]. Disponível em: <http://www.healthierus.gov/ dietaryguidelines>. Acesso em 26 jul. 2005.

[23] Karanja NM, Obarzanek E, Lin PH, McCullough ML, Phillips KM, Swain JF et al. Descriptive characteristics of the dietary patterns used in the Dietary Approaches to Stop Hypertension trial. JADA 1999; 99 (8 Suppl 1): 19-27.

[24] [SPSS] Statistical Package for the social Sciences (SPSS) for windows Student (software). Versão 10. Chicago: Marketing Department; 1996.

[25] Garcia GCB, Gambardella AMD, Frutuoso MFP. Estado nutricional e consumo alimentar de adolescentes de um centro de juventude da cidade de São Paulo. Rev Nutr 2003; 16 (1): 41-50.

[26] Carvalho CMRG, Nogueira AMP, Teles JBM, Paz SMR, Sousa RML. Consumo alimentar de adolescentes matriculados em um colégio particular de Teresina, Piauí, Brasil. Rev Nutr 2001; 14 (2): 85-93.

[27] Bulck JV, Mierlo JV. Energy intake associated with television viewing in adolescents, a cross sectional study. Appetite 2004; 43 (2):181-4.

[28] Epstein LH, Roemmich JN, Paluch RA, Raynor HA. Influence of changes in sedentary behavior on energy and macronutrient intake in youth. Am J Clin Nutr 2005; 81 (2): 361-6.

[29] Sichieri R. Epidemiologia da obesidade. Rio de Janeiro: UERJ; 1998.

[30] Stockman NKA, Schenkel TC, Brown JN, Duncan AM. Comparison of energy and nutrient intakes among meals and snacks of adolescents males. Prev Med 2005; 41 (1): 203-10.

[31] Albano RD. Estado nutricional e consumo alimentar de adolescentes [dissertação de mestrado]. São Paulo: Universidade de São Paulo; 2000.

[32] Lerner BR, Lei DLM, Chaves SP, Freire RD. O cálcio consumido por adolescentes de escolas públicas de Osasco, São Paulo. Rev Nutr 2000; 13 (1): 57-63.

[33] Speck BJ, Bradley CB, Harrell JS, Belyea MJ. A Food Frequency Questionnaire for Youth: Psychometric Analysis and Summary of Eating Habits 
in Adolescents. J Adolesc Health 2001; 28:16-25.

[34] Demory-Luce D, Morales M, Nicklas T, Baranowski T, Zakeri I, Berenson G. Changes in Food Group Consumption Patterns from Childhood to Young Adulthood: The Bogalusa Heart Study. JADA
2004; 104 (11):1684-91.

[49] MAGAREY A, Daniels LA, Smith A. Fruit and vegetable intakes of Australians aged 2-18 years: an evaluation of the 1995 National Nutrition Survey data. Aust N Z J Public Health 2001; 25 (2): 155-61. 